\title{
NUCLEAR MAGNETIC RESONANCE STUDIES OF GLUCOSE METABOLISM IN NON-INSULIN-DEPENDENT DIABETES MELLITUS SUBJECTS
}

\author{
Robert G. Shulman, Ph.D. \\ Department of Molecular Biophysics and Biochemistry, \\ Yale University, New Haven, Connecticut, U.S.A.
}

In vivo nuclear magnetic resonance (NMR) measurements have allowed accurate determination of glycogen concentrations and rates of synthesis, measured by ${ }^{13} \mathrm{C}$ NMR, and of glucose-6phosphate (G6P) concentrations, measured by

${ }^{31} \mathrm{P}$ NMR, in non-insulin-dependent diabetes mellitus (NIDDM) subjects and their age/weightmatched controls. These NMR measurements, when combined with more standard biochemical data, have provided important insights into the metabolic basis of NIDDM. The present review summarizes NMR studies carried out in our laboratory. These have been presented as simply as possible by ignoring the complexities of the experimental methods and conditions, and without recounting the significance of other studies which have been properly addressed in our original publications.

\section{THE ROLE OF MUSCLE GLYCOGENESIS IN GLUCOSE DISPOSAL}

NIDDM is a genetic disease afflicting about $5 \%$ of the American population. In "closed societies," such as the Pima Indians in Arizona, most adults are stricken by NIDDM (1). The disease is characterized by hyperglycemia (high plasma glucose levels) with numerous, presumably secondary, pathological conditions in the eyes, kidneys, and other organs. NIDDM patients produce insulin from the $\beta$ islets of their pancreas; they are, however, resistant to the hormone's action and

Address correspondence and reprint requests to: Robert G. Shulman, Yale University, MR Center, P.O. Box 208043, New Haven, CT 06520-8043, U.S.A. have slower glucose disposal rates than normal individuals. In early life, insulin resistance is counterbalanced by insulin overproduction by the pancreas; as a result, while young subjects often have impaired glucose tolerance, they are not substantially hyperglycemic. The disease develops later in life when insulin secretion no longer compensates for resistance. This "switch" depends upon body weight, diet, and exercise in ways not yet completely understood.

At the time we began our NMR experiments, studies had indicated that, after a glucose load, glucose was consumed mainly in the muscle, where it was presumably stored as glycogen (2). It was thus believed that muscle was the principal site of insulin resistance in NIDDM. Measurements of the rate of muscle glycogen synthesis, however, were not sufficiently accurate to test this hypothesis directly. Our contribution to these studies consisted of providing accurate measurements of glycogen concentrations both in muscle and in liver by using ${ }^{13} \mathrm{C}$ NMR. Glycogen in solution (3) and in vivo (4) gave wellresolved resonances in the ${ }^{13} \mathrm{C}$ NMR spectra, which, when compared with those from standardized solutions, allowed quantitative determination of glycogen concentrations in vivo (5). This was unexpected for a large molecule like glycogen (molecular weight: $\sim 10^{8}$ daltons) that ordinarily gives resonances too broad to be resolved. Subsequent chemical NMR experiments, however, showed that the glycogen molecule has uniformly rapid internal motion, thereby explaining its spectral visibility.

The experiments reviewed here were all performed in a 2.1-T magnet $1 \mathrm{~m}$ in diameter. Subjects were placed in the magnet by lying supine 


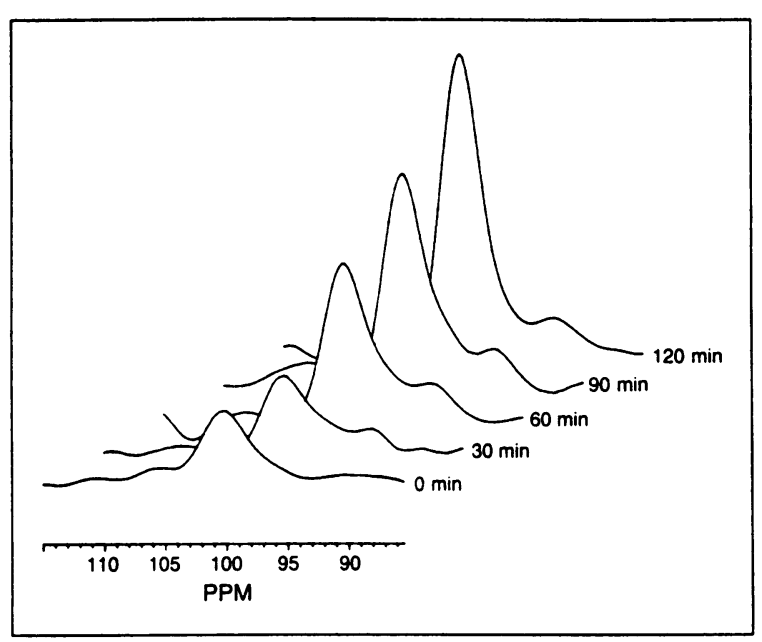

FIG. 1. Typical course of ${ }^{13} \mathrm{C}$ NMR spectra of muscle glycogen in a representative normal subject during a hyperglycemic-hypersulinemic clamp

The $\mathrm{Cl}$ peak of glycogen appears at $100.4 \mathrm{ppm}$. Adapted from Shulman et al., N. Engl. J. Med. 322: 223-228. Copyright 1990. Massachusetts Medical Society. All rights reserved.

on a sliding bed. The Bruker NMR spectrometer was significantly modified in order to obtain high-resolution images and ${ }^{1} \mathrm{H},{ }^{13} \mathrm{C}$, or ${ }^{31} \mathrm{P}$ NMR spectra. NMR spectra were obtained from a circular surface coil $9 \mathrm{~cm}$ in diameter tuned either to the ${ }^{13} \mathrm{C}$ or ${ }^{31} \mathrm{P}$ frequencies. Proton decoupling, imaging, and shimming were accomplished by a larger concentric coil tuned to the ${ }^{1} \mathrm{H}$ resonance frequency. Spectra were obtained by a simple pulse-acquire sequence, while decoupling was usually achieved by continuous wave irradiation.

In our first experiments, ${ }^{13} \mathrm{C}$ NMR measurements of muscle glycogen concentrations were obtained in subjects with NIDDM and in their age/weight-matched controls during a hyperinsulinemic-hyperglycemic clamp, which simulated post-prandial conditions (Fig. 1) (6). In both groups, total muscle glycogen synthesis was equal to total nonoxidative glucose consumption (Fig. 2). The two groups, however, differed in their rates of muscle glycogen synthesis (and the corresponding rates of nonoxidative glucose consumption), where NIDDM patients synthesized glycogen one-half as fast as controls. This experiment thus indicated that reduced muscle glycogen synthesis is the major factor responsible for the deficit in glucose disposal in NIDDM.

The reduced muscle glycogen synthesis in subjects with NIDDM could represent a defect either present before developing symptoms of

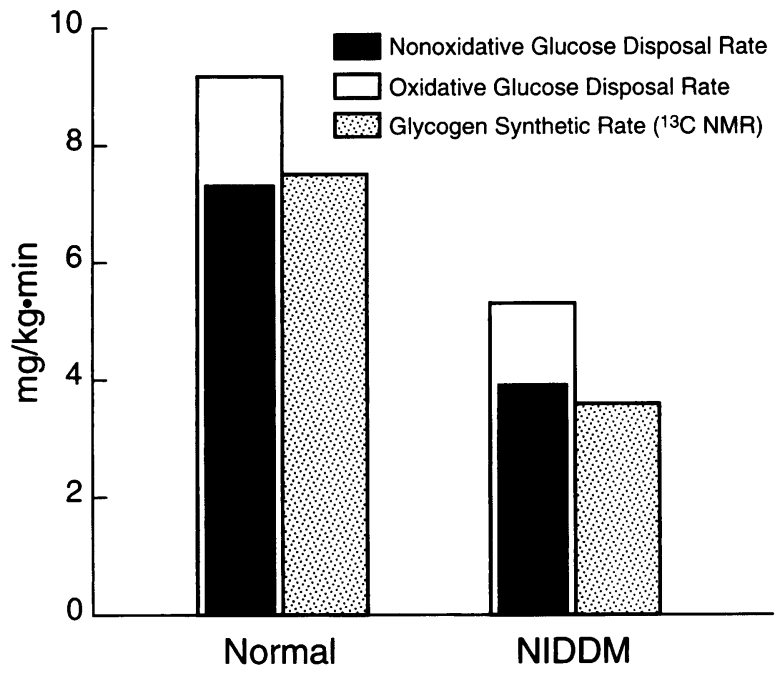

FIG. 2. Oxidative and nonoxidative glucose disposal and glycogen synthesis rates in normal and IDDM subjects

The oxidative and nonoxidative glucose disposal rates were determined from glucose clamp requirements and indirect calorimetry. The whole body rate of glycogen synthesis was calculated by adding the ${ }^{13} \mathrm{C}$ NMR rate measured in the gastrocnemius muscle over the total body muscle mass. In both groups, total nonoxidative glucose disposal is accounted for by total muscle glycogen synthesis, but in the NIDDM group this rate is about one-half of that in the control group. Adapted from Shulman et al., $N$. Engl. J. Med. 322: 223-228. Copyright 1990. Massachusetts Medical Society. All rights reserved.

the disease or result from the down-regulation of one or more steps in the pathway by chronic hyperglycemia. In order to answer this question, we performed the identical experiment in young healthy subjects who were classified as being at high risk of developing diabetes (prediabetics) on the basis of having diabetic parents and impaired glucose tolerance (7). Similar to subjects with NIDDM, the glycogen synthesis rate of prediabetics was one-half that of the controls. These results provided strong evidence that the metabolic defect in NIDDM was indeed in the muscle glycogen synthesis pathway and that it was genetic in origin.

\section{MUSCLE GLUCOSE TRANSPORTERS/HEXOKINASE AND THE SITE OF INSULIN RESISTANCE}

Figure 3 shows the well-established muscle glycogen synthesis pathway. During stimulation by insulin or when recovering from glycogen de- 


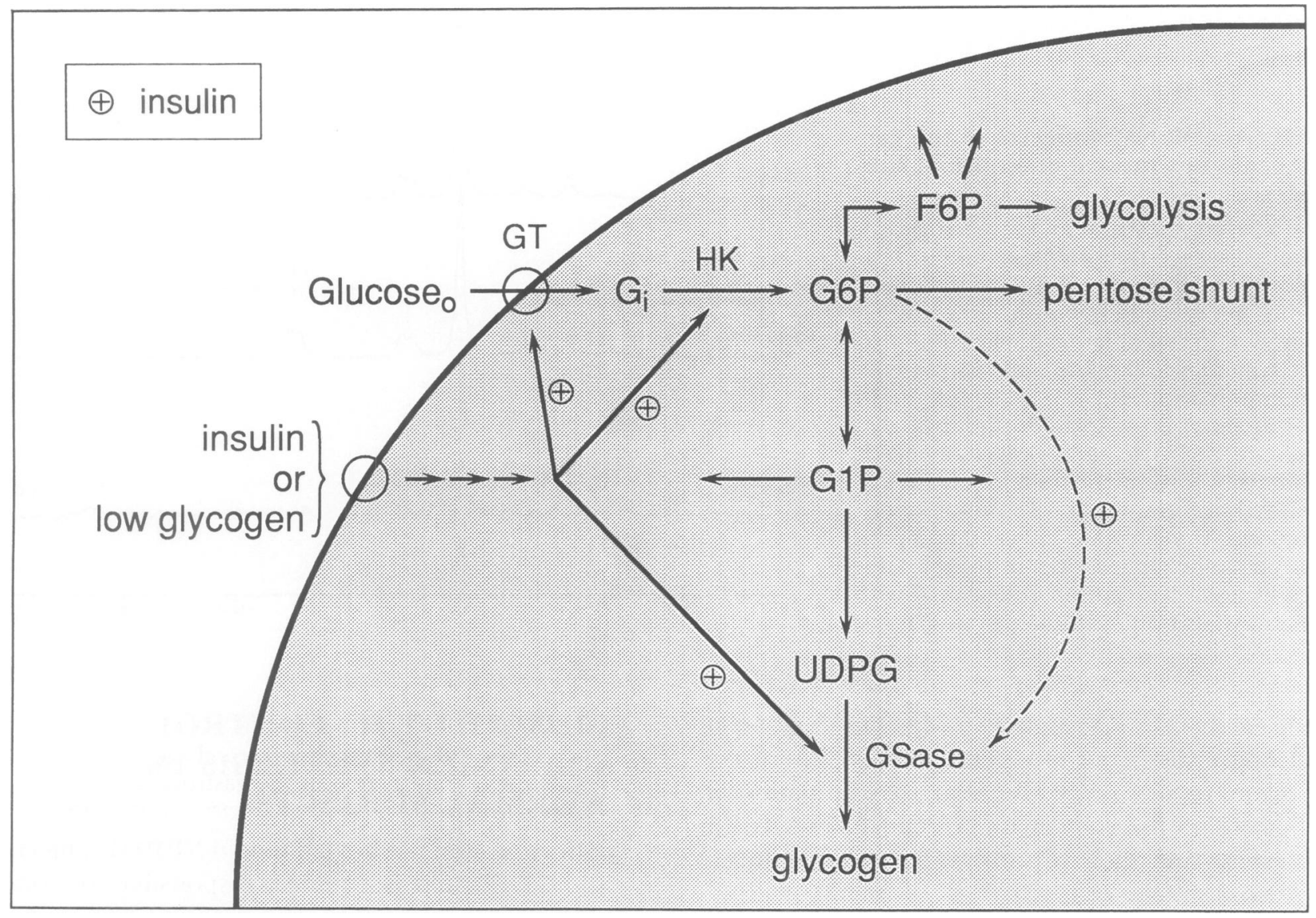

FIG. 3. Schematic representation of the glycogenic pathway

It shows intermediates, branching points, and some hormonal control points. G6P, glucose-6-phosphate; GT, glucose transporter; $\mathrm{Hk}$, hexokinase; $\mathrm{G}_{\mathrm{i}}$, intracellular glucose.

pleting exercise, glucose transporters are recruited from intracellular vesicles to the plasma membrane $(8,9)$. This, in turn, facilitates diffusion of glucose into the cell, where it is converted to G6P. Insulin also controls another step in this pathway: the activity of the enzyme muscle glycogen synthase (GSase). This enzyme has four identical subunits each containing nine serines that can be phosphorylated and dephosphorylated by numerous kinases and phosphatases, thereby regulating its level of activity $(10,11)$. Since GSase is activated synchronously with changes in glycogenic flux, the enzyme has often been described as "controlling" the rate of glycogen synthesis. If indeed GSase activity were the rate determining step in glycogen synthesis, one might expect that G6P concentrations in NIDDM patients (who have reduced glycogen synthesis rates) would be higher than in normal controls under the same clamp conditions.
As we had previously been able to resolve the G6P resonance by ${ }^{31} \mathrm{P}$ NMR in the human gastrocnemius muscle (12), we believed it would be possible to quantitate steady-state G6P concentrations during the hyperglycemic-hyperinsulinemic clamp. The results of this experiment are shown in Fig. 4. Contrary to expectations, muscle G6P concentrations were lower in NIDDM patients than in normal controls during the same clamp conditions. Since the driving force for glycogen synthesis was the same for both groups, this indicated that the defect in the glycogen synthesis pathway in NIDDM patients is proximal to G6P, implicating either glucose transporters (GT) or hexokinase (Hk) activity.

This conclusion, although unexpected, was not completely surprising, since both GT and Hk enzyme activities are controlled by insulin. As mentioned above, recruitment of GT to the plasma membrane after insulin stimulation has been well characterized (8); similarily, increased 
FIG. 4. ${ }^{31} \mathrm{P}$ NMR spectra of the gastrocnemius muscle of a normal subject

A baseline spectrum and a spectrum acquired over 40-80 min into the lowdose insulin clamp, as well as the difference between these two spectra, are shown. In the difference spectrum (clamp minus baseline), and increase in the resonances of $\mathrm{G} 6 \mathrm{P}$ ( $7.13 \mathrm{ppm})$ and $\mathrm{Pi}$ $(4.88 \mathrm{ppm})$ is observed during the clamp. The increase in G6P resonance intensity corresponds to an increase in G6P concentration of $0.13 \mathrm{mmol} / \mathrm{kg}$ of muscle. The decrease in Pcr (0.00 ppm) is not completely shown in this plot due to its greater magnitude. Reprinted with permission from Rothman et al. (7).

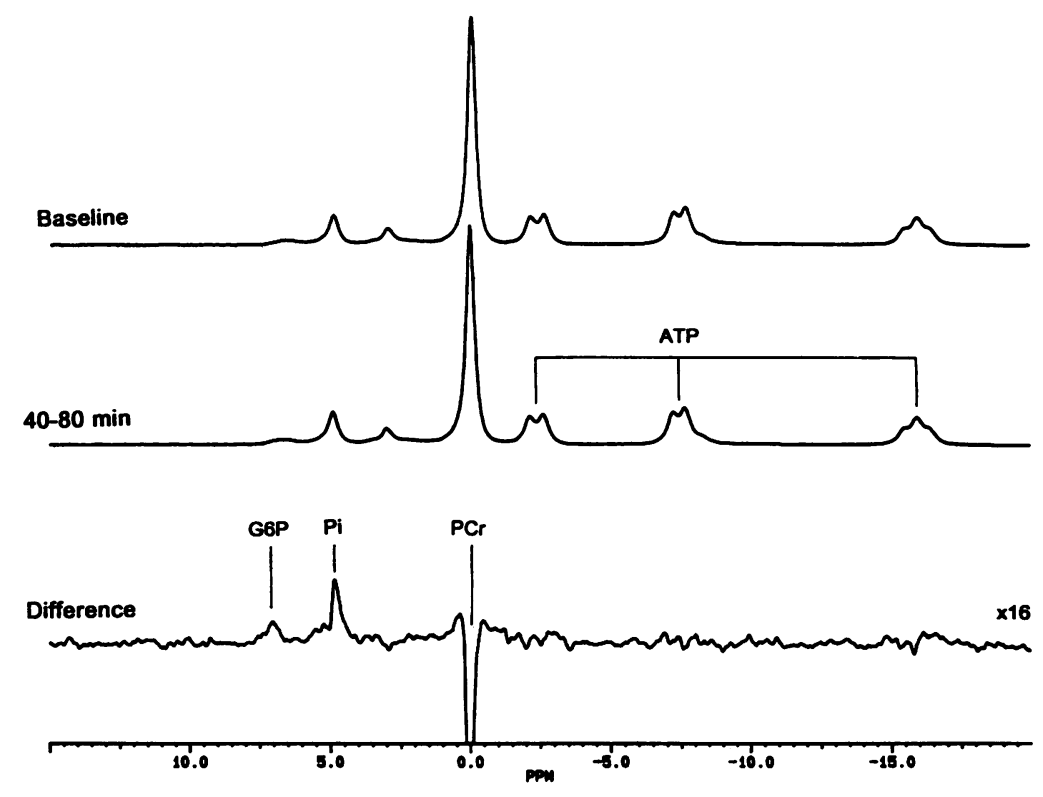

Hk activity in response to insulin has been strongly suggested (13). Furthermore, there are several reports suggesting that the stimulation of glucose transport by insulin is reduced in NIDDM patients and their offsprings, based on reduced glucose uptake $(14,15)$.

Despite this evidence indicating that reduced GT/Hk activity is responsible for reduced glucose consumption in NIDDM, the notion that GSase activity "controls" glycogenic flux in normal individuals has persevered. Surprisingly, experimental data provided little support for this view. Prior to our NMR studies, there was poor agreement between muscle GSase activity measured in human muscle biopsy samples and in vivo glycogenic flux. It was known from the early work of Piras and colleagues (16) that G6P is a positive allosteric effector of GSase. Their experiments showed that in vivo concentrations of ATP inhibited GSase and that this inhibition was overcome by G6P concentrations varying between $\sim 100 \mu \mathrm{M}$ for the more active form of GSase to several millimolar for the less active form. However, difficulties in correlating GSase activities measured in human biopsy samples with flux had been confounded by G6P concentrations reported in biopsies which were too high to provide agreement with glucose uptake measurements (presumably due to extraction artifacts) (17). Our noninvasive measurements of G6P allowed us to show that in the rat muscle (where we could assay the enzyme activity accurately) there was excellent agreement between GSase activity and the flux.

\section{MUSCLE GT/HK CONTROL GLYCOGEN SYNTHESIS IN NORMAL SUBJECTS}

Our experiments showed that in NIDDM subjects the GT/Hk step was mainly responsible for controlling glycogenic flux. This did not prove, however, that this early step also controlled flux in healthy subjects since the primary defect in NIDDM could have caused a reduction in GT/Hk activity. For this reason, experiments were designed in normal human and rat muscle in accordance with the metabolite control analysis (MCA) developed by Kacser and colleagues (18). They had proposed that each step in a pathway can be characterized by a flux control coefficient (C), where $C$ is the fractional change in flux/ fractional change in enzyme, while maintaining all the other parameters constant. In a linear pathway, the maximum value of this coefficient is one for a particular step, which would indicate that it controls the flux. Our experiments consisted of altering GT/Hk enzyme activity by changing plasma glucose levels while maintaining insulin concentrations constant; the rate of glycogen synthesis was then measured by ${ }^{13} \mathrm{C}$ NMR (7). This showed that flux was proportional to plasma glucose concentrations and established a flux control coefficient of approximately one for the GT/Hk step (19). Thus, a model was proposed in which flux control in muscle glycogen synthesis pathway is primarily at the GT/Hk (and not the GSase) step. According to this model, increased glucose transport raises G6P levels, 
which in turn increase GSase activity by allosteric control, thereby ensuring agreement between GSase activity and flux.

Given that the GT/Hk step controls the rate of glycogen synthesis, why is the activity of GSase so tightly regulated by phosphorylation? The simple answer is that GSase activity is regulated in order to avoid unphysiologically high levels of G6P during changes in flux. This novel function of enzyme phosphorylation to control levels of an intermediate in a pathway, rather than controlling flux, provides alternative explanations for the many kinases and phosphatases identified in biochemical pathways and these possibilities are further discussed elsewhere (20).

\section{GLYCOGEN SYNTHESIS AFTER EXERCISE}

Intense exercise depletes glycogen and leads to a subsequent period of resynthesis (21). Early biopsy measurements (22) indicated that after intense depletion, a rapid period of synthesis occurs during the first hour, followed by several hours of slower synthesis and gradual return to basal levels. In studies where exogenous insulin was administered to subjects with insulin-dependent diabetes mellitus, the rapid phase was shown to be insulin independent and the subsequent phase insulin dependent (22).

These results have been confirmed and extended by our in vivo ${ }^{13} \mathrm{C}$ NMR studies of healthy nondiabetic subjects. Rates of glycogen synthesis were derived from the slope of the curve obtained by plotting glycogen concentrations as a function of time (23). The rapid phase of glycogen resynthesis was observed only after severe glycogen depletion ( $<25 \%$ of basal) and the period of slower resynthesis after less severe depletion (Fig. 5). The transition from insulin-independent to insulin-dependent phases took place at $\sim 30 \mathrm{mM}$ glucose equivalents. The insulindependent and -independent phases were identified by measurements using somatostatin infusion to inhibit insulin secretion. In the absence of insulin, the initial rapid phase was not affected, while the slower, later phase was completely inhibited (23).

We then studied the effects of insulin resistance by determining glycogen synthesis rates after exercise that decreased muscle glycogen to $\sim 25 \%$ of basal levels in eight young, lean, normoglycemic, insulin-resistant offspring of par-

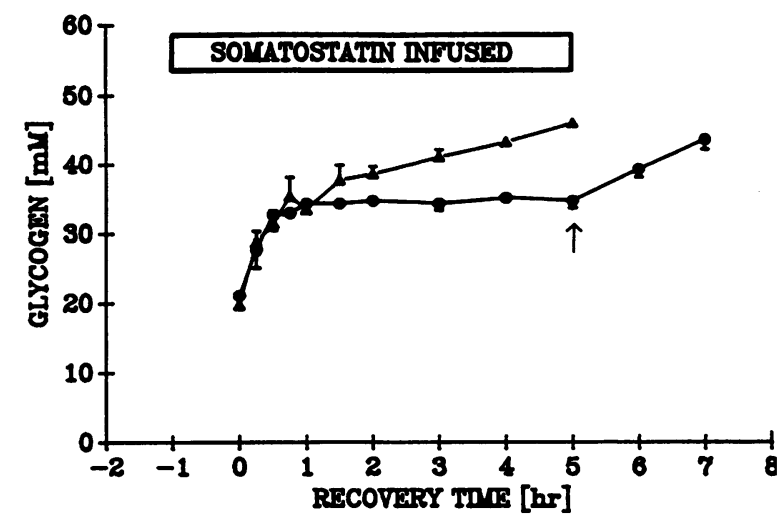

FIG. 5. Recovery of glycogen after depletion to $25 \%$ of resting levels in normal subjects under conditions where insulin secretion is either normal or inhibited by somatostatin infusion

- conditions in which insulin secretion was inhibited; $\boldsymbol{\Delta}$, normal insulin secretion; $\uparrow$, return of insulin secretion to normal (somatostatin infusion was discontinued). Reprinted with permission from Price et al. (23).

ents with NIDDM and compared them with those in age/weight-matched controls $(24,25)$. Glycogen synthesis rates during the first hour after exercise, which is the insulin-independent recovery period, was the same in both groups (15.5 $\pm 1.3 \mathrm{mM} / \mathrm{hr}$ in insulin-resistant subjects and $15.8 \pm 1.7 \mathrm{mM} / \mathrm{hr}$ in the controls). After the first hour, during the insulin-dependent period, glycogen synthesis rates in the insulin-resistant subjects were negligible but they were significantly higher in the controls. These results provide evidence that the early, rapid rate of glycogen resynthesis after glycogen-depleting exercise is not impaired in the insulin-resistant offspring of NIDDM parents but the later, insulin-dependent phase is considerably impaired.

\section{NMR STUDIES OF HEPATIC GLYCOGEN IN NIDDM SUBJECTS AND CONTROLS}

Since the ${ }^{13} \mathrm{C}$ isotope measured in these NMR experiments is only $1.1 \%$ naturally abundant, in our studies signal strength was sometimes improved by infusing subjects with substrates containing ${ }^{13} \mathrm{C}$ (such as $1-{ }^{13} \mathrm{C}$ glucose). This type of labeling also permitted the measurement of turnover rates and thus to distinguish a kinetically inert glycogen pool from one that is continually being synthesized and degraded. In the 
muscle, our experiments showed that the glycogen pool at rest was static. After infusion with $1-{ }^{13} \mathrm{C}$ glucose and intense exercise of one leg, turnover of label was not detected in the nonexercised leg. In contrast, in the liver glycogen was continually being synthesized and degraded, even at steady-state concentrations (26). This was established by monitoring glycogen concentrations by ${ }^{13} \mathrm{C}$ NMR during $1-{ }^{13} \mathrm{C}$ glucose infusion in the rat and chasing with unlabeled glucose.

In a series of experiments using fed and fasted healthy nondiabetic subjects, we showed that the rate of hepatic glycogen degradation, or glycogenolysis, increased with higher glycogen concentrations. In a complementary fashion, the rate of glycogen synthesis decreased as glycogen concentrations increased. Thus, the resting level of hepatic glycogen is in a dynamic steady state where synthesis equals degradation (27).

It is well known that the rate of hepatic glucose production is higher in diabetics. In order to determine the individual contributions of gluconeogenesis and glycogenolysis to hepatic glucose production, we used ${ }^{13} \mathrm{C}$ NMR methods. Experiments were conducted first in a group of nondiabetic human subjects in order to establish the methodology and then repeated in another cohort of subjects with NIDDM and their age/ weight-matched controls. Measurements taken in the first experiments (28) on young normal subjects during a 68-hr fast, were based on a model whereby hepatic glucose production is equal to gluconeogenic flux plus glycogenolytic flux. Rates of total glucose production were calculated by infusing $6-{ }^{3} \mathrm{H}$-labeled glucose during the fast and measuring the decrease in radioactivity as unlabeled glucose was produced. Glycogenolysis rates were determined by measuring hepatic glycogen by ${ }^{13} \mathrm{C}$ NMR as a function of time (Fig. 6). In order to measure total hepatic glycogen, volumetric measurements by magnetic resonance imaging (MRI) were taken several times during the fast to correct the ${ }^{13} \mathrm{C}$ NMR intensities which measured glycogen concentrations by changes in liver volume. Gluconeogenesis accounted for $64 \pm 5 \%$ of total glucose production during the first $22 \mathrm{hr}$ of the fast. In the subsequent 14- and 18-hr periods, gluconeogenesis accounted for $82 \pm 5 \%$ and $96 \pm 1 \%$, respectively, of total glucose production. Based on these in vivo measurements, gluconeogenesis accounted for a larger fraction of glucose production than had been previously reported.

In a similar study using NIDDM patients in

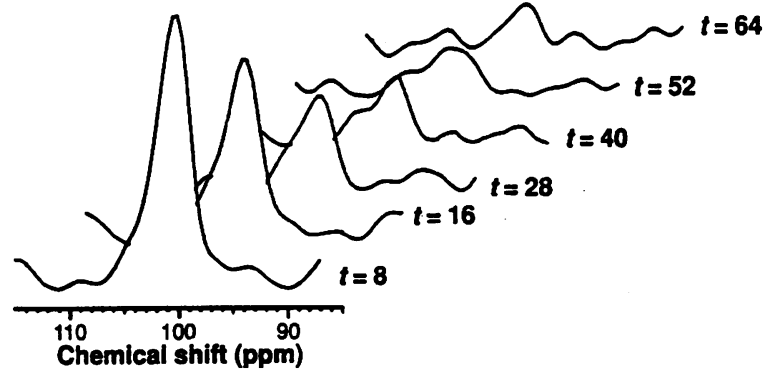

FIG. 6. Typical course of ${ }^{13} \mathrm{C}$ NMR spectra of the naturally abundant $\mathrm{Cl}$ position of hepatic glycogen in a representative normal subject during $64 \mathrm{hr}$ of fasting

Reprinted with permission from Rothman et al., Science 254: 573-576. Copyright 1991. American Association for the Advancement of Science.

the first $23 \mathrm{hr}$ of fasting, gluconeogenesis was shown to account for $88 \pm 2 \%$ of glucose production in the NIDDM patients and $70 \pm 6 \%$ in their age/weight-matched controls. We concluded that gluconeogenesis is the pathway responsible for the majority of glucose production both in NIDDM patients and in normal controls and that the increased rate of hepatic glucose production in NIDDM patients is due to enhanced gluconeogenesis.

\section{SUMMARY}

In this review, the results of a series of NMR experiments investigating glucose storage and synthesis in NIDDM patients and normal controls have been summarized. These have shown:

1. The deficit in nonoxidative glucose disposal in NIDDM subjects results from a defect in the muscle glycogen synthesis pathway.

2. Reduced activity of glucose transporter/ hexokinase step in this pathway accounts for the reduced rate of glycogen synthesis in NIDDM patients.

3. This reduced activity of GT/Hk is a genetic defect present before the clinical onset of disease in prediabetic descendants of diabetic parents.

4. In muscle from normal, healthy subjects the rate of glycogen synthesis is controlled by the glucose transport/hexokinase activity step and not by the activity of the muscle glycogen synthase enzyme. 
5. Hepatic gluconeogenesis is responsible for most hepatic glucose production during an overnight fast in both normal and NIDDM subjects, and increases in gluconeogenic flux are responsible for the increased rate of hepatic glucose production in NIDDM subjects.

6. In contrast to human muscle, where glycogenesis ceases at rest, in the liver gluconeogenesis and glycogenolysis are always active.

Numerous previous studies were considered prior to embarking in each of these NMR experiments. In the original research articles we published, the earlier studies were discussed in terms of the relevant literature. Here, however, I have chosen to present the NMR data as simply as possible, in the hope of exposing the significance of these studies by disentangling the results from the complexities of NMR methodology.

\section{ACKNOWLEDGMENTS}

These results have been collaborative throughout, and my colleagues are mentioned in the references. I would particularly like to acknowledge the contributions of Professors D. L. Rothman and Gerald I. Shulman.

\section{REFERENCES}

1. Bogardus C, Lillioja S, Howard BV, Reaven G, Mott D. (1984) Relationship between insulin secretion, insulin action, and fasting plasma glucose concentration in nondiabetic and non-insulin-dependent diabetic subjects. J. Clin. Invest. 74: 1238-1246.

2. DeFronzo RA, Ferrannini E, Simonson DC. (1989) Fasting hyperglycemia in non-insulin dependent diabetes mellitus: Contribution of excessive hepatic glucose production and impaired tissue glucose uptake. Metab. Clin. Exp. 38: 387-395.

3. Sillerud LO, Shulman RG. (1983) Structure and metabolism of mammalian liver glycogen monitored by ${ }^{13} \mathrm{C}$ NMR. Biochemistry 22: 1087-1094.

4. Jue T, Rothman DL, Tavitian BA, Shulman RG. (1989) Natural abundance ${ }^{13} \mathrm{CNMR}$ study of glycogen repletion in human liver and muscle. Proc. Natl. Acad. Sci. U.S.A. 86: 1439-1442.
5. Gruetter R, Prolla TA, Shulman RG. (1991) ${ }^{13} \mathrm{C}$ NMR visibility of rabbit muscle glycogen in vivo. Magn. Res. Med. 20: 327-332.

6. Shulman GI, Rothman DL, Jue T, Stein P, DeFronzo RA, Shulman RG. (1990) Quantitation of muscle glycogen synthesis in normal subjects and subjects with non-insulin dependent diabetes by ${ }^{13} \mathrm{C}$ nuclear magnetic resonance spectroscopy. $N$. Engl. J. Med. 322: 223-228.

7. Rothman DL, Magnusson I, Cline G, et al. (1995) Decreased muscle glucose transport/ phosphorylation is an early defect in the pathogenesis of non-insulin dependent diabetes mellitus. Proc. Natl. Acad. Sci. U.S.A. 92: 983-987.

8. Doven AG, Ramlal T, Rostogi S, et al. (1990) Exercise induces recruitment of the "insulinresponsive glucose transporter." J. Biol. Chem. 265: 13427-13430.

9. Wallberg-Henriksson $\mathrm{H}$, Constable $\mathrm{SH}$, Young DA, Holloszy JO. (1988) Glucose transport into rat skeletal muscle: Interaction between exercise and insulin. J. Appl. Physiol. 65: 909-913.

10. Roach PJ, Larner J. (1976) Rabbit skeletal muscle glycogen synthase II: Enzyme phosphorylation state and effector concentrations as interacting control parameters. J. Biol. Chem. 251: 1920-1925.

11. Harris RA. (1992) Carbohydrate metabolism 1: Major metabolic pathways and their control. In: Devlin TM (ed). Textbook of Biochemistry with Clinical Correlations. Wiley-Liss, New York, pp. 343-351.

12. Pan JW, Hamm JR, Rothman DL, Shulman RG. (1989) In-vitro titration of phosphomonoesters by ${ }^{1} \mathrm{H}$ decoupled ${ }^{31} \mathrm{P}$ NMR in human skeletal muscle after exercise. In: $A b$ stract, Soc. Magn. Reson. Med., 8th Annual Meeting. Amsterdam p. 542.

13. Lueck JD, Fromm HJ. (1975) Kinetics, mechanism, and regulation of rat skeletal muscle hexokinase. J. Biol. Chem. 249: 2259_ 2268.

14. Pendergrass J, Fazioni E, Saccomani MP, Collins D, Giovannia G. (1995) In vivo glucose transport and phosphorylation in skeletal muscle is impaired in insulin resistant, normal glucose tolerant offspring of two NIDD parents. Abstract in Diabetes 44: 197.

15. Bonadonna RC, Del Prato S, Saccomani MP, et al. (1993) Transmembrane glucose transport in skeletal muscle of patients with non- 
insulin dependent diabetes. J. Clin. Invest. 92: 486-494.

16. Piras R, Rothman LB, Cabib E. (1968) Regulation of muscle glycogen synthetase by metabolites. Differential effects of the I and D forms. Biochemistry 7: 56-66.

17. Harris RC, Hultman E, Nordesjo L-O. (1974) Glycogen, glycolytic intermediates and high energy phosphates determined in biopsy samples of musculus quadriceps femoris of man at rest: methods and variance of values. Scand. J. Clin. Invest. 33: 109-120.

18. Kacser H, Burns JA. (1973) The control of flux. Symp. Soc. Exp. Biol. 27: 65-104.

19. Shulman RG, Bloch G, Rothman DL. (1995) In vivo regulation of muscle glycogen synthase and the control of glycogen synthesis. Proc. Natl. Acad. Sci. U.S.A. 92: 8535-8542.

20. Shulman RG, Rothman DL. (1996) Enzymatic phosphorylation of muscle glycogen synthase: A mechanism for maintenance of metabolic homeostasis. Proc. Natl. Acad. Sci. U.S.A. 93: 7491-7495.

21. Richter EA, Garetto LP, Goodman MN, Ruderman NB. (1984) Enhanced muscle glucose metabolism after exercise: Modulation by local factors. Am. J. Physiol. 246: E476E482.

22. Maehlum S, Hostmark AT, Hermansen L. (1977) Synthesis of muscle glycogen during recovery after severe exercise in diabetic and non-diabetic subjects. Scand. J. Clin. Invest. 37: 309-316.
23. Price TB, Rothman DL, Taylor R, Shulman GI, Avison MJ, Shulman RG. (1994) Human muscle glycogen resynthesis after exercise: Insulin-dependent and independent phases. J. Appl. Physiol. 76: 104-111.

24. Price TB, Perseghin G, Duleba A, et al. (1996) NMR studies of muscle glycogen synthesis in insulin resistant offspring of NIDDM parents immediately following glycogen depleting exercise. Proc. Natl. Acad. U.S.A. 93: 5329-5334.

25. Shulman RG, Rothman DL, Price TB. (1996) NMR studies of muscle and applications to exercise and diabetes. Diabetes 45: S93-S98.

26. David M, Petit WA, Laughlin MR, Shulman RG, King JE, Barrett EJ. (1990) Simultaneous synthesis and degradation of rat liver glycogen: An in vivo NMR spectroscopic study. J. Clin. Invest. 86: 612-617.

27. Magnusson I, Rothman DL, Jucker B, Cline GW, Shulman RG, Shulman GI. (1994) Liver glycogen turnover in fed and fasted humans. Am. J. Physiol. 266: E796-E803.

28. Rothman DL, Magnusson I, Katz LD, Shulman RG, Shulman GI. (1991) Quantitation of hepatic glycogenolysis and gluconeogenesis in fasting humans using ${ }^{13} \mathrm{CNMR}$. Science 254: 573-576.

29. Magnusson I, Rothman DL, Katz LD, Shulman RG, Shulman GI. (1992) Increased rate of gluconeogenesis in type II diabetes mellitus. A ${ }^{13} \mathrm{C}$ nuclear magnetic resonance study. J. Clin. Invest. 90: 1323-1327. 